\title{
G protein signaling in the parasite Entamoeba histolytica
}

\author{
Dustin E Bosch ${ }^{1}$ and David P Siderovski ${ }^{2}$
}

The parasite Entamoeba histolytica causes amebic colitis and systemic amebiasis. Among the known amebic factors contributing to pathogenesis are signaling pathways involving heterotrimeric and Ras superfamily G proteins. Here, we review the current knowledge of the roles of heterotrimeric G protein subunits, Ras, Rho and Rab GTPase families in E. histolytica pathogenesis, as well as of their downstream signaling effectors and nucleotide cycle regulators. Heterotrimeric $\mathrm{G}$ protein signaling likely modulates amebic motility and attachment to and killing of host cells, in part through activation of an RGS-RhoGEF (regulator of G protein signaling-Rho guanine nucleotide exchange factor) effector. Rho family GTPases, as well as RhoGEFs and Rho effectors (formins and p21-activated kinases) regulate the dynamic actin cytoskeleton of $E$. histolytica and associated pathogenesis-related cellular processes, such as migration, invasion, phagocytosis and evasion of the host immune response by surface receptor capping. A remarkably large family of 91 Rab GTPases has multiple roles in a complex amebic vesicular trafficking system required for phagocytosis and pinocytosis and secretion of known virulence factors, such as amebapores and cysteine proteases. Although much remains to be discovered, recent studies of $G$ protein signaling in $E$. histolytica have enhanced our understanding of parasitic pathogenesis and have also highlighted possible targets for pharmacological manipulation.

Experimental \& Molecular Medicine (2013) 45, e15; doi:10.1038/emm.2013.30; published online 22 March 2013

Keywords: amebic colitis; Entamoeba histolytica; G proteins; GTPases; RGS proteins

\section{ENTAMOEBA HISTOLYTICA CAUSES AMEBIC COLITIS AND SYSTEMIC AMEBIASIS}

Epidemiology, disease sequelae and current treatment options

The parasite E. histolytica is the causative agent of infectious amebic colitis and systemic amebiasis. ${ }^{1}$ The worldwide prevalence of E. histolytica infection is not precisely known, with the most recent published estimates ${ }^{2}$ being approximately 50 million infections and 100000 deaths annually. Epidemiological estimates have been historically complicated by limitations of diagnostic tests, as well as difficulty in differentiating E. histolytica from the morphologically similar but typically non-pathogenic related Entamoeba species, E. dispar and E. moshkovskii. ${ }^{3}$ However, more recently developed antigen detection and PCR-based modalities with improved sensitivity and specificity have allowed more accurate regional estimations of E. histolytica infections. ${ }^{4,5}$ The prevalence of E. histolytica infection is particularly high among susceptible populations with limited access to clean water. For instance, a study of preschool-aged children in Bangladesh revealed annual infections in $40-50 \%$ of subjects, ${ }^{6}$ a profile of Orang Asli ethnic groups in Malaysia found an overall prevalence of E. histolytica-positive stool samples to be 15-20\%, ${ }^{7}$ and E. histolytica was detected by PCR in $10-15 \%$ of a rural Mexican population. ${ }^{8}$ The prevalence of antibodies specific for E. histolytica in sera of a Chinese population varied from 0.5 to $14 \%$, depending on geographical location. ${ }^{9}$ An inter-relationship between host nutritional status and susceptibility to E. histolytica infection has also recently begun to emerge (reviewed in Verkerke et al. ${ }^{10}$ ). Although E. histolytica infection is relatively rare in developed countries, such as the United States, it does occur among travelers, immigrants and select susceptible subpopulations. ${ }^{11,12}$ Furthermore, outbreaks of E. histolytica have occurred due to contaminated municipal water supplies (for example, Barwick et al. ${ }^{13}$ ).

The life cycle of E. histolytica consists of an interchange between an encysted form and a motile, pathogenic,

\footnotetext{
${ }^{1}$ Department of Pharmacology, The University of North Carolina at Chapel Hill, Chapel Hill, NC, USA and ${ }^{2}$ Department of Physiology and Pharmacology, West Virginia University School of Medicine, Robert C. Byrd Health Sciences Center, Morgantown, WV, USA

Correspondence: Dr DP Siderovski, Department of Physiology and Pharmacology, 3051A Health Sciences Center, West Virginia University School of Medicine, Morgantown, WV 26506-9229, USA.

Email: dpsiderovski@hsc.wvu.edu
}

Received 27 January 2013; accepted 3 February 2013 
trophozoite form. E. histolytica cysts, shed in the feces of infected human hosts, are transmitted primarily by ingestion of contaminated water or food. ${ }^{1}$ Excystation occurs in the small intestine, and the resultant E. histolytica trophozoites may then colonize the large intestine while evading the host immune response. ${ }^{3}$ Although the majority of E. histolytica infections are asymptomatic, trophozoites can penetrate the intestinal mucous barrier, resulting in colitis. ${ }^{1}$ Amebic colitis is characterized by trophozoite-mediated killing of intestinal epithelial cells and responding immune cells, as well as local tissue destruction. ${ }^{14}$ In rare cases, E. histolytica trophozoites can enter the blood stream and spread systemically, giving rise to abscesses, primarily in the liver and less frequently in the lungs and brain. ${ }^{3}$ Although systemic amebiasis requires previous intestinal infection, amebic liver abscesses can develop in the absence of symptomatic colitis ${ }^{14,15}$ and are known to appear months or years following exposure. ${ }^{16}$ Thus, treatment is recommended for patients with E. histolytica infection, even in the absence of symptomatic disease. ${ }^{3}$

Nitroimidazoles, such as metronidazole, are the current best drugs for the treatment of invasive amebiasis. ${ }^{3}$ Approximately $90 \%$ of patients with mild or moderate amebic colitis respond to nitroimidazole therapy, although persistent intestinal infection often requires additional treatment with paromomycin or diloxanide furoate for complete eradication. ${ }^{3}$ However, a significant fraction of patients with E. histolytica infection do not respond to nitroimidazoles, and relatively rare side effects such as allergic reactions, neuropathies and additional gastrointestinal symptoms can also affect treatment tolerance. ${ }^{17}$ Resistance of E. histolytica infection to nitroimidazoles and paromomycin has not yet emerged as a major limitation to treatment; however, numerous examples of antibiotic resistance in other microorganisms warrants further exploration of alternative pharmacological therapeutics. ${ }^{18}$ A recent study identified auranofin, an FDA (Food and Drug Administration)-approved rheumatoid arthritis drug, as a potent inhibitor of E. histolytica thioredoxin reductase and further demonstrated its protective effects in a mouse model of amebic colitis. ${ }^{19}$ Other classes of compounds have also recently been pursued as nanomolar-potency inhibitors of E. histolytica growth in culture. ${ }^{20,21}$ Despite existing effective therapies, E. histolytica infection and associated disease remains endemic in many parts of the world, particularly in areas with contaminated drinking water and food sources. ${ }^{6,8}$ Problems with sanitation implementation and access to appropriate therapeutics could potentially be circumvented by the development of an E. histolytica vaccine, and efforts toward this goal are ongoing (for example, Abd Alla et al. ${ }^{22}$ ).

\section{Parasite factors in pathogenesis}

A number of E. histolytica molecular components have been thoroughly established as contributors to its pathogenesis. During initial intestinal colonization, E. histolytica adheres to the colonic mucin layer primarily through a galactoseinhibitable lectin, known as the Gal/GalNAc lectin (reviewed in Petri et al. ${ }^{23}$ ). The trimeric surface protein is also a dominant factor in parasite attachment to host cells and subsequent tissue destruction and functions interdependently with the dynamic actin cytoskeleton of E. histolytica. ${ }^{23}$ Trophozoites also secrete pore-forming peptides known as 'amebapores' that assemble within host cell membranes to trigger cell death (reviewed in Leippe and $\mathrm{Herbst}^{24}$ ). A relatively large family of $E$. histolytica-encoded cysteine proteases also contributes to host cell killing, as well as degradation of the host extracellular matrix during invasive amebic infection and evasion of the host immune response through proteolysis of immunoglobulins and complement (reviewed in Que and Reed ${ }^{25}$ ). Many regulators of the actinrich cytoskeleton within E. histolytica are also emerging as contributors to pathogenesis-related processes, such as phagocytosis of host cells, trophozoite motility and tissue invasion, and shedding of host antibodies by surface receptor capping (reviewed in Voight and Guillen, ${ }^{26}$ Tavares et al. ${ }^{27}$ Meza et al. ${ }^{28}$ and Labruyere and Guillen ${ }^{29}$ ).

\section{HETEROTRIMERIC G PROTEINS AND RAS SUPERFAMILY GTPASES}

Sequencing of the complete E. histolytica genome $^{30}$ and genome-wide expression studies (for example, Gilchrist et $a .^{31}$ ) have revealed large numbers of putative cell signaling molecules expressed in this single-celled parasite, including a substantial family of $>300$ kinases. ${ }^{32}$ Also prominent within the $E$. histolytica genome are genes encoding heterotrimeric $G$ protein subunits $(G \alpha, G \beta$ and $\mathrm{G} \gamma$ ) and a large number of small, $\sim 21 \mathrm{kDa} G$ proteins belonging to the Ras superfamily. ${ }^{30} \mathrm{G} \alpha$ subunits and Ras GTPases are molecular switches and cellular signaling nodes that bind guanine nucleotides (guanosine triphosphate (GTP) or guanosine diphosphate (GDP)) through highly conserved, nucleotide-interacting sequencing motifs. ${ }^{33,34}$ As mammalian $\mathrm{G}$ proteins are known to be master regulators of cellular functions spanning cell division and proliferation, cytoskeletal dynamics, vesicular trafficking and specific responses to extracellular cues, ${ }^{33,35}$ it is likely that E. histolytica homologs are similarly important for trophozoite biology and pathogenicity. G protein signaling pathways are also notable for their amenability to pharmacological manipulation; particularly, heterotrimeric $G$ protein signaling via $\mathrm{G}$ protein-coupled receptors (GPCRs) is the target of approximately one-third of all currently FDAapproved drugs. ${ }^{36,37}$

\section{REGULATION OF THE GUANINE NUCLEOTIDE CYCLE Heterotrimeric $\mathrm{G}$ proteins}

A $\mathrm{G} \alpha$ subunit in its inactive, GDP-bound state forms a heterotrimer with the obligate $\mathrm{G} \beta \gamma$ dimer (Figure 1). A seven-transmembrane domain GPCR, when activated by an extracellular ligand, engages the heterotrimer and catalyzes the release of GDP by the G $\alpha$ subunit. ${ }^{38}$ Thus the GPCR is a guanine nucleotide exchange factor (GEF) for the G $\alpha$ subunit, promoting GDP release and subsequent binding of GTP, which is present in a higher concentration than GDP in the 
cytoplasm. $^{34}$ Nucleotide exchange is accompanied by structural rearrangement of three switch regions in the Ras-like domain of the $G \alpha$ subunit, resulting primarily from nucleotide-binding pocket interactions with the $\gamma$-phosphoryl group of GTP. ${ }^{39}$ The activated G $\alpha$-GTP separates from the $\mathrm{G} \beta \gamma$ dimer, and both components are free to signal through various downstream effectors. ${ }^{34}$ Mammalian $G \alpha$ subtypes engage different effectors: $G \alpha_{s}$ activates, while $G \alpha_{i / o}$ inhibits, cyclic AMP (cAMP) generation by adenylyl cyclase; $\mathrm{G} \alpha_{\mathrm{q}}$ stimulates phospholipase- $\mathrm{C} \beta$ activity and subsequent release of intracellular calcium stores; and $\mathrm{G} \alpha_{12 / 13}$ signaling leads to Rho GTPase activation through RhoGEFs. ${ }^{34,40}$ Signaling is terminated by the intrinsic GTPase activity of the G $\alpha$ subunit, leading to release of free phosphate and repeated formation of the $\mathrm{G} \alpha \cdot \mathrm{GDP} / \beta \gamma$ heterotrimer (Figure 1). G $\alpha$ subunitmediated GTP hydrolysis, and thus signal termination, is accelerated by a family of GTPase-accelerating proteins (GAPs) known as 'regulators of $\mathrm{G}$ protein signaling' (RGS proteins). ${ }^{41}$ RGS proteins do not directly contribute to the GTP hydrolysis reaction, but instead stabilize the $\mathrm{G} \alpha$ switch regions to allow
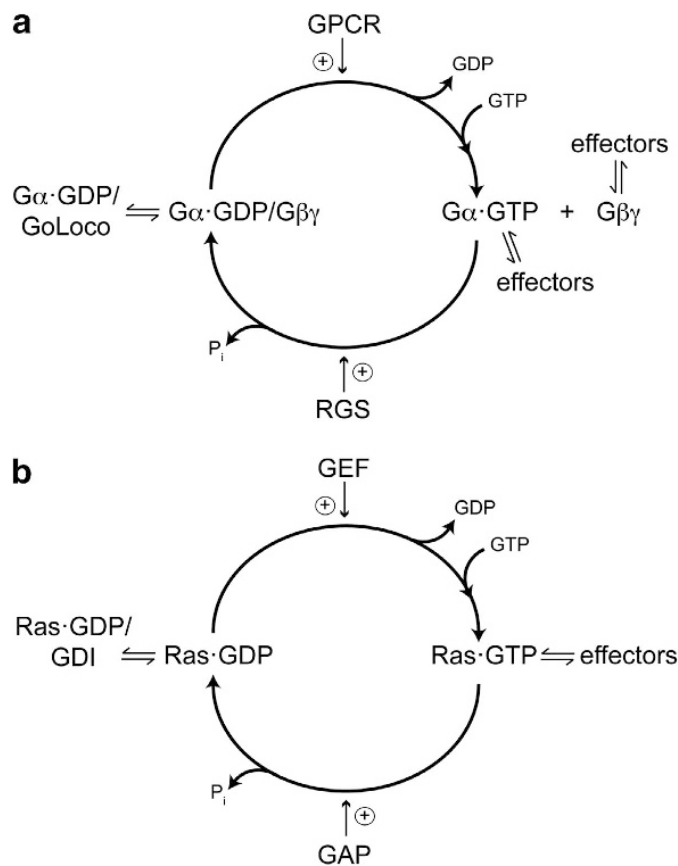

Figure 1 Nucleotide cycle regulation of heterotrimeric and Ras superfamily $\mathrm{G}$ proteins. (a) $\mathrm{G} \alpha$ subunits cycle between guanosine diphosphate (GDP)- and guanosine triphosphate (GTP)-bound states. G protein-coupled receptors (GPCRs) serve as guanine nucleotide exchange factors (GEFs) for $G$ protein heterotrimers, stimulating their release of GDP. Conversely, GoLoco motifs are guanine nucleotide dissociation inhibitors (GDIs) that slow nucleotide exchange by $\mathrm{G} \alpha$ subunits. RGS proteins are GTPaseaccelerating proteins (GAPs) for $\mathrm{G} \alpha$ subunits, promoting signal termination by both activated $\mathrm{G} \alpha$ subunits and free $\mathrm{G} \beta \gamma$. (b) The small $G$ protein nucleotide cycle parallels that of heterotrimeric $G$ proteins, with GEF-stimulated and GDI-inhibited nucleotide exchange as well as GAP-mediated activation of GTP hydrolysis. $R G S$, regulators of $G$ protein signaling. for efficient hydrolysis. ${ }^{42}$ Some $\mathrm{G} \alpha$ subunit effectors also enhance GTPase activity; particularly, phospholipase-C $\beta$ serves as a GAP for $G \alpha_{q}$, and the $G \alpha_{12 / 13}$ subfamily RGSRhoGEF effectors possess a GTPase-accelerating domain (the rgRGS domain) with distant homology to RGS proteins. ${ }^{40,43}$ An additional class of $\mathrm{G} \alpha$ regulators is the GoLoco motif protein family, members of which serve as guanine nucleotide dissociation inhibitors (GDIs) by binding directly to G $\alpha$-GDP and preventing nucleotide release. ${ }^{44}$

\section{Ras superfamily GTPases}

The nucleotide cycle of Ras superfamily $G$ proteins closely parallels that of heterotrimeric G proteins. Inactive, GDPbound Ras GTPases are activated by guanine nucleotide exchange factors (GEFs) in a process that involves structural rearrangement of two switch regions within the $G$ protein to promote release of GDP and the $\mathrm{Mg}^{2+}$ cofactor (Figure 1)..$^{33,45}$ Following binding of GTP, activated Ras superfamily GTPases engage a host of different downstream effectors. In contrast to heterotrimeric $G$ proteins, the intrinsic GTPase activity of Ras superfamily members is typically very slow. Thus, Ras superfamily-specific GAPs truly 'activate' GTP hydrolysis (rather than merely accelerate hydrolysis as is the case with G $\alpha$ GAPs) by contributing directly to the reaction, as typified by the 'arginine finger' of p120GAP. ${ }^{46,47}$ In another distinct difference with $\mathrm{G} \alpha$ subunits, Ras superfamily GTPases typically possess a C-terminal cysteine residue that is isoprenylated in cells by specific lipid moiety transferases, a post-translational modification that promotes their membrane association..$^{33}$ GDIs associated with Ras superfamily GTPases slow nucleotide exchange and employ an isoprenyl group-binding site to extract GTPases from, and shuttle them between, cellular membranes. ${ }^{48,49}$

\section{HETEROTRIMERIC G PROTEIN SIGNALING IN E. HISTOLYTICA}

Before completion of the E. histolytica genome sequencing project, ${ }^{30}$ indirect evidence for heterotrimeric $G$ protein signaling components existing within E. histolytica accumulated in the literature, but specific genes and associated protein products had not been identified. Studies on the effects of histamine and serotonin, typical GPCR agonists, on E. histolytica trophozoites revealed alterations in pathogenicity and phagocytic activity, as well as enhancement of virulence in a mouse model, ${ }^{50-53}$ suggesting the possible presence of a hormone-sensing $G$ protein signaling pathway within E. histolytica. Exposure of E. histolytica to fibronectin fragments was seen to result in actin cytoskeleton rearrangements, as well as changes in intracellular calcium and cAMP levels, ${ }^{54-56}$ raising the possibility of fibronectinresponsive $G \alpha_{q}, G \alpha_{s}$ and/or $G \alpha_{i / o}$ signaling within trophozoites. Additional indirect evidence arose from studies utilizing cholera toxin (CTX) and pertussis toxin (PTX), factors known to adenosine diphosphate (ADP)-ribosylate and activate $G \alpha_{s}$ or inhibit $G \alpha_{i / o}$ signaling, respectively. ${ }^{57}$ Both CTX and PTX were reported to ADP-ribosylate 
multiple proteins of diverse molecular weights in trophozoite lysates, and toxin treatment led to increased cAMP formation in both cytoplasmic and cell membrane preparations, as well as increased adhesion to a fibronectin-coated surface. ${ }^{58}$ Studies in the related species Entamoeba invadens further suggested the possibility of heterotrimeric $G$ protein signaling in Entamoeba. The catecholamines epinephrine and norepinephrine, classical GPCR agonists in mammals, were found to promote $E$. invadens encystation at high-nanomolar to low-micromolar concentrations, although a traditional concentration-response pattern was not observed. ${ }^{59}$ The authors hypothesized the presence of a $\beta 1$ adrenergic receptor-like molecular entity on trophozoite cell surfaces, as further supported by radioligand binding with a specific antagonist. Furthermore, chromatography techniques identified catecholamines within E. histolytica extracts, suggesting a potential autocrine $G$ protein signaling loop. ${ }^{59}$ Additional studies implied that CTX or PTX treatment, as well as the adenylyl cyclase-stimulating compound forskolin, could also promote cAMP accumulation in (and encystation of) E. invadens, while application of an adenylyl cyclase inhibitor was reported to have opposite effects. ${ }^{60}$ Together with epinephrine-induced binding of GTP $\gamma S$ on trophozoite membranes, these findings were suggestive of an adrenergic receptor signal transduction cascade within Entamoeba involving $G \alpha_{\mathrm{s}^{-}}$and/or $G \alpha_{\mathrm{i} / \mathrm{o}^{-}}$-like proteins, with opposing regulatory effects on an adenylyl cyclase.

However, the sequenced E. histolytica genome, ${ }^{30}$ as well as those of E. dispar and E. invadens, have revealed only the presence of two putative $G \alpha$ subunits, a single $G \beta$ subunit and at least two $G \gamma$ subunits. ${ }^{61,62}$ Absent from the genome are clear homologs to mammalian phospholipase- $\mathrm{C} \beta$ as well as $\mathrm{G}$ protein-regulated adenylyl cyclases or cyclic nucleotide phosphodiesterases. ${ }^{30}$ Thus, although exposure of E. histolytica to stimuli such as fibronectin and catecholamines may result in cAMP accumulation or increased intracellular calcium levels, it is unlikely that these observed effects are mediated by traditional $\mathrm{G} \alpha_{\mathrm{s}}$ /adenylyl cyclase, $\mathrm{G} \alpha_{\mathrm{i} / \mathrm{o}}$ /adenylyl cyclase or $\mathrm{G} \alpha_{\mathrm{q}} /$ phospholipase-C $\beta$ signaling pathways. Also, we have been unable to identify within the E. histolytica genome any genes encoding clear homologs of adrenergic, histamine or serotonin GPCRs (unpublished data and Bosch et al. ${ }^{61}$ ), suggesting that the functional effects of these biogenic amines on trophozoites may not be mediated by traditional GPCR/heterotrimeric $\mathrm{G}$ protein signal transduction.

Analysis of both the sequence and structure of the $\mathrm{G} \alpha$ subunit EhG $\alpha 1$ revealed a lack of homology to mammalian $\mathrm{G} \alpha$ subfamilies, including $\mathrm{G} \alpha_{\mathrm{s}}$ and $\mathrm{G} \alpha_{\mathrm{i} / \mathrm{o}}{ }^{61}$ This finding, together with a lack of the $\mathrm{C}$-terminal cysteine required for ADP ribosylation by PTX, suggests that EhG $\alpha 1$ is unlikely to be specifically modified by bacterial toxins. ${ }^{61}$ The observed effects of CTX and PTX treatment on Entamoeba trophozoites might instead result from off-target effects, a hypothesis supported by CTX- and PTX-mediated ADP ribosylation of multiple proteins of diverse molecular weights in E. histolytica trophozoite lysates. ${ }^{58}$ Despite its lack of phylogenetic relationship to any particular mammalian $\mathrm{G} \alpha$ subfamily, ${ }^{61}$ $\mathrm{EhG} \alpha 1$ shares functional similarity with mammalian $\mathrm{G}_{12 / 13}$ subunits in engaging and contributing to the activation of an RGS-RhoGEF effector (Figure 2). ${ }^{63}$ An evolutionary origin of E. histolytica heterotrimeric $\mathrm{G}$ protein signaling independent from, but functionally convergent with, that of mammalian $\mathrm{G} \alpha_{12 / 13} /$ RGS-RhoGEF pathways is suggested by multiple factors, including the sequence and structural divergence of EhGal, the canonical nature of its interaction with the EhRGS-RhoGEF RGS domain (that is, as opposed to the rgRGS domain found in mammalian RhoGEFs), and the structural features of the autoinhibited EhRGS-RhoGEF. ${ }^{61,63}$ Expression of constitutively active EhG $\alpha 1$ and EhRacC mutants, together with the effector EhRGS-RhoGEF, leads to Rho family GTPase activation in Drosophila S2 cells, ${ }^{63}$ suggesting that heterotrimeric $G$ protein and Rho family GTPase signaling pathways communicate in E. histolytica (Figure 2). However, no specific Rho family GTPase has yet been identified as an EhRGS-RhoGEF substrate. Overexpression of either wild-type $E h G \alpha 1$ or a dominant-negative, constitutively EhG $\beta \gamma$-bound EhG $\alpha 1$ mutant has opposing effects on trophozoite migration, invasion, and host cell attachment and killing, suggesting that heterotrimeric G protein signaling modulates multiple pathogenesis-related behaviors. ${ }^{61}$ Perturbation of EhG $\alpha 1$ expression also leads to significant changes in the E. histolytica transcriptome and alters the secretion of cytotoxic cysteine proteases, ${ }^{61}$ suggesting a possible functional overlap with Rab family GTPases (see below). Overexpression of EhRGS-RhoGEF has similar

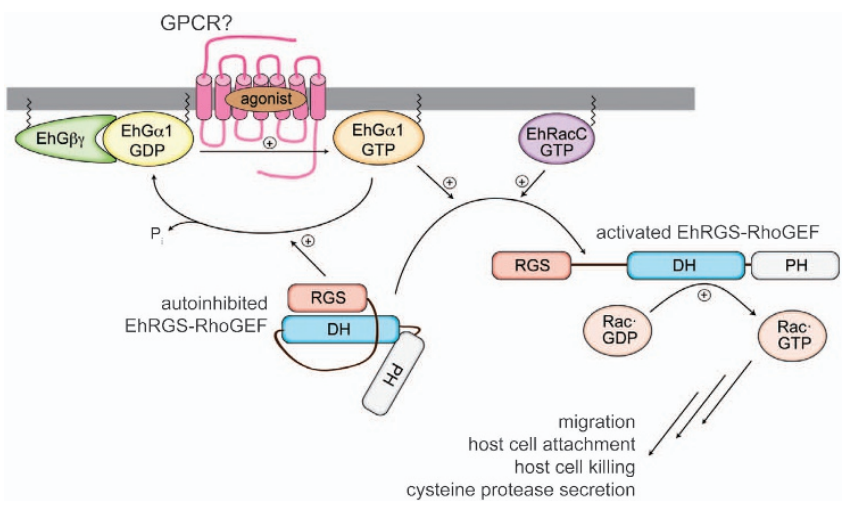

Figure 2 Model of heterotrimeric $G$ protein signaling in E. histolytica. Activated $E h G \alpha 1$, together with guanosine triphosphate (GTP)-bound EhRacC, engages the autoinhibited EhRGS-RhoGEF ( $E$. histolytica regulator of $\mathrm{G}$ protein signaling-Rho guanine nucleotide exchange factor) to promote Rac GTPase in Drosophila S2 cells, ${ }^{63}$ although no specific E. histolytica Rho family substrate for EhRGS-RhoGEF has yet been identified. Both EhG $\alpha 1$ and EhRGS-RhoGEF alter trophozoite migration, host cell attachment and cell killing by altered cysteine protease secretion. ${ }^{61,63}$ An associated G protein-coupled receptor (GPCR) is postulated but not yet established within this signaling pathway; Despite its name, the protein EhGPCR-1 is more likely a Wntbinding factor than a ligand-activated heterotrimeric $G$ protein GEF. ${ }^{61} \mathrm{DH}$, Dbl homology; GDP, guanosine diphosphate; $\mathrm{PH}$, pleckstrin homology. 
effects on trophozoite function when compared with overexpression of dominant-negative EhG $\alpha 1$, consistent with the function of EhRGS-RhoGEF as a EhG $\alpha 1$ GAP (also demonstrated in vitro) and, thus, a negative regulator of heterotrimeric $G$ protein signaling in the context of its overexpression. ${ }^{61,63}$ Nucleotide exchange is rate-limiting in the EhG $\alpha 1$ nucleotide cycle, ${ }^{61}$ as seen with mammalian $G \alpha$ subunits, suggesting that GEF activity is needed for signal activation. Yet the E. histolytica genome lacks homologs of non-receptor GEFs for heterotrimeric G proteins such as Ric-8 and $\mathrm{GIV},{ }^{30,64}$ leading to the hypothesis that E. histolytica may express one or more GPCRs (that is, a putative cell surfacespanning, EhG $\alpha 1$-directed GEF; Figure 2). Although a bona fide heterotrimeric GPCR has not yet been identified in this organism, one or more receptor/ligand pairs would provide valuable tools for manipulating $G$ protein signal transduction in E. histolytica and also potentially serve as a candidate drugdiscovery target. ${ }^{36,61}$

A second, putative $\mathrm{G} \alpha$ subunit (AmoebaDB acc. no. EHI_186910) exhibits a unique domain structure, with an $\mathrm{N}$-terminal $\mathrm{G} \alpha$-like fold easily identifiable despite substantial sequence divergence from mammalian $\mathrm{G} \alpha$ subunits and a C-terminal PP2C-related phosphatase domain. ${ }^{62}$ The G $\alpha$-like region of this protein also lacks determinants for CTX- or PTX-mediated ribosylation (as does EhG $\alpha 1$ ); furthermore, this putative $G \alpha$ subunit also lacks the otherwise very wellconserved nucleotide-binding motifs shared among all $G$ proteins, suggesting a lack of nucleotide binding by this protein (unpublished data and Wittinghofer and Vetter ${ }^{65}$ ). This apparently expressed protein awaits functional assessment of its $G \alpha$-like domain and its unique relationship to the adjacent phosphatase domain.

EhG $\beta 1$ dimerizes with one of two E. histolytica $\mathrm{G} \gamma$ subunits when expressed in mammalian cells, and the EhG $\beta \gamma$ dimer in turn binds EhG $\alpha 1$ in a nucleotide state-selective fashion. ${ }^{61}$ $\mathrm{G} \beta \gamma$ subunits also frequently engage downstream effectors, even when the associated $\mathrm{G} \alpha$ subunits lack a major known effector, as seen in the cases of Arabidopsis thaliana sugar-sensing and yeast pheromone signaling. ${ }^{66,67}$ Signaling downstream of EhG $\beta \gamma$ is a distinct possibility for E. histolytica and may contribute to the phenotypic effects of perturbed EhG $\alpha 1$ expression; ${ }^{61}$ however, no EhG $\beta \gamma$ effectors have yet been identified.

\section{RAS SUPERFAMILY GTPASES IN E. HISTOLYTICA}

The $E$. histolytica genome encodes a remarkably large number of small GTPases for a single-celled parasite ( $>170$ annotated in $A$ moebaDB ${ }^{68}$ ), suggesting a prominent role for Ras superfamily $G$ protein signaling. The Ras superfamily can be divided into the Ras subfamily, typically regulating cell proliferation and survival; the Rho family that regulates actin organization, the cell cycle and gene expression; the Ran family, implicated primarily in nucleocytoplasmic transport; and the Rab and Arf families, known as regulators of vesicular transport and trafficking (reviewed in Wennerberg et al. ${ }^{33}$ ). Ten Ras proteins and two related Rap homologs have been described in E. histolytica, ${ }^{69,70}$ although the complete Ras subfamily has not been enumerated since completion of the E. histolytica genome sequencing project. At least 20 Rho family GTPases, including Rho, Rac and Cdc42 homologs, are transcribed by E. histolytica trophozoites. ${ }^{71-73}$ The Rab family is the most numerous small $G$ protein group described in E. histolytica, with 91 annotated genes. ${ }^{74}$ Although not yet described in the literature, putative Ran and Arf family GTPases also exist in the E. histolytica genome. ${ }^{68}$ Although a small fraction of E. histolytica Ras superfamily GTPases has been investigated, the extent of functional redundancy, signaling specificity and nucleotide cycle regulation among these small G proteins remain largely unknown. Given the poor genetic tractability of E. histolytica trophozoites, investigations of $G$ protein signaling in this organism have largely been limited to overexpression studies. Although overexpression is certainly an informative genetic perturbation, it should be noted that overexpressed G proteins, or nucleotide cycle-impaired mutants thereof, are subject to potential mislocalization and non-physiological functions.

\section{Ras family GTPases}

An initial study in E. histolytica trophozoites identified two Ras genes and two related Rap genes, as well as a single protein that apparently cross-reacted with a mammalian anti-Ras antibody. ${ }^{69}$ Ras family GTPases in mammals and yeast are isoprenylated with either a geranylgeranyl or a farnesyl group at the characteristic C-terminal CaaX motif, where ' $a$ ' is an aliphatic amino acid, and the final residue is predictive of either geranylgeranylation or farnesylation. ${ }^{33}$ Expression of EhRap2, EhRas1 and CaaX motif mutants thereof in mammalian reticulocytes revealed that E. histolytica Ras GTPases can be isoprenylated, but that their CaaX motif sequences are less predictive of the specific isoprenyl group added than mammalian counterparts. ${ }^{75}$ An E. histolytica farnesyltransferase, consisting of two subunits, was later cloned and shown to farnesylate human H-Ras and EhRas4 to the exclusion of three other E. histolytica Ras isoforms, indicating a distinct CaaX motif selectivity for isoprenylation. ${ }^{70}$ Recombinant $E$. histolytica farnesyl transferase is resistant to mammalian farnesyltransferase inhibitors, precluding their use as tools in studying Ras GTPase function in E. histolytica trophozoites. Ras GTPases and related signaling machinery have been the targets of much pharmaceutical development effort, given the centrality of oncogenic Ras signaling to cellular proliferation and survival in many human malignancies. ${ }^{76}$ However, no studies of perturbed Ras signaling in E. histolytica have yet emerged. Similarly, putative regulators of Ras nucleotide cycling (for example, GEFs and GAPs) and candidate Ras effectors are currently understudied in E. histolytica.

\section{Rho family GTPases}

E. histolytica possesses a highly dynamic, actin-rich cystoskeleton that participates in many pathogenesis-related processes (reviewed in Meza et al. ${ }^{28}$ ), as well as two major 
actin-associated myosins (reviewed in Labruyere and Guillen ${ }^{29}$ and Marion et al. ${ }^{77}$ ). Remarkably rapid actin remodeling is apparent in trophozoite motility, ${ }^{78}$ a process regulated by extracellular matrix interactions ${ }^{79}$ as well as self-generated chemokines. ${ }^{80}$ Cytoskeletal remodeling is also intimately associated with $E$. histolytica phagocytosis ${ }^{26}$ and surface receptor capping. ${ }^{27}$ As master regulators of the actin cytoskeleton, as well as cell division and transcription in mammals, Rho GTPases and their associated proteins have been a focus of intense investigation in E. histolytica.

The first identified Rho family GTPase in E. histolytica was EhRho1 (Figure 3a), also later referred to as EhRhoA1. ${ }^{71}$ As a homolog of human RhoA, EhRhol was a natural candidate substrate for the Rho-inhibiting C3 exoenzyme from Clostridum botulinum, a protein whose ectopic expression in E. histolytica trophozoites leads to ribosylation of an $\sim 25-\mathrm{kDa}$ protein and reduces both proliferation and host cell killing. ${ }^{81}$ However, recombinant EhRhol was later found not to be a substrate for C3 exoenzyme, ${ }^{82}$ but instead glucosylated in vitro by Clostridum difficile toxin B and Clostridum novyi $\alpha$-toxin. ${ }^{83}$ However, use of these two Clostridium toxins to study EhRho1 function in vivo is impaired by a lack of trophozoite membrane permeability. ${ }^{83} \mathrm{~A}$ structural study of EhRhol has more recently highlighted its conserved conformational difference between GDP- and GTP-bound states, as well as its distinct lack of a 'Rho insert helix'-a structural feature that differentiates all other Rho family GTPases from the greater Ras superfamily. ${ }^{73}$ EhRho1 also differs from its mammalian homologs at a key nucleotide-binding residue, a feature found to confer rapid intrinsic nucleotide exchange but not constitutive activity. ${ }^{73,82}$ However, EhRho1 does exhibit a signature activity of other Rho family GTPases; expression of a constitutively active mutant in human cells promotes actin stress fiber formation. ${ }^{73}$ Activated EhRho1.GTP binds a Diaphanous-related formin effector protein, EhFormin1, to the exclusion of other E. histolytica Rho family GTPases (Figure 3a). ${ }^{73,84}$ EhForminl is known to modulate actin polymerization, to be autoinhibited by an $\mathrm{N}$ - to C-terminal intramolecular interaction like its well-studied mammalian homologs, ${ }^{85}$ and to be specifically activated by EhRho1.GTP. ${ }^{84}$ A recent crystal structure of the EhRho1·GTP $\gamma$ S/EhFormin1 complex revealed a similar mode of intermolecular interaction as compared with mammalian counterparts, with the exception of a missing secondary binding site involving the Rho insert helix, and further yielded insights into specificity requirements for Rho GTPase/effector pairings. ${ }^{84}$ EhFormin1 (also called EhDia) belongs to a family of eight E. histolytica formin proteins, three of which are Diaphanous-related (that is, containing tandem Rho GTPase-binding domains (GBDs) and formin homology 3 domains (FH3s)). ${ }^{86,87}$ Overexpressed EhFormin1 in trophozoites localizes to pseudopodia, the microtubular assembly in the nucleus, and cytoplasmic F-actin structures in response to serum. ${ }^{87}$ Furthermore, EhFormin1- and EhFormin2-overexpressing ameba exhibit cell division defects, with an increased number of nuclei per
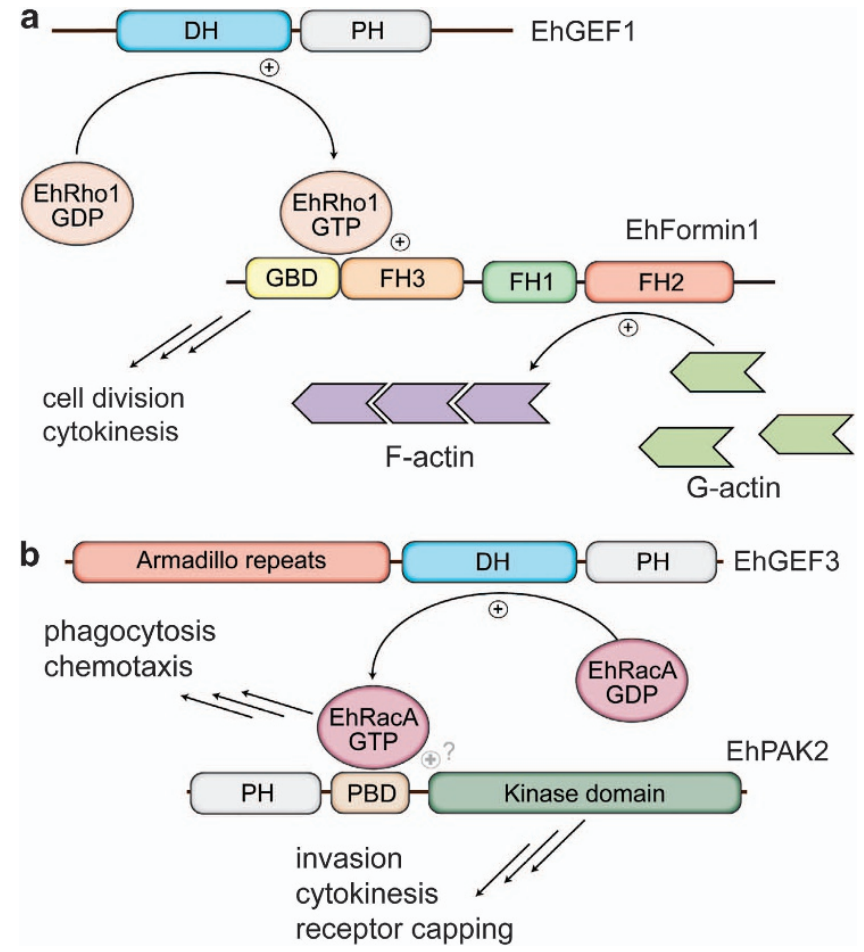

Figure 3 EhRhol and EhRacA signaling modulate pathogenic behaviors in E. histolytica. (a) Nucleotide exchange on EhRhol is known to be catalyzed by $E$. histolytica guanine nucleotide exchange factor 1 (EhGEF1) in vitro. ${ }^{98}$ EhRho1 engages the GTPase-binding domain-formin homology 3 (GBD-FH3) domain tandem of the diaphanous-related and autoinhibited EhFormin 1 to modulate actin polymerization. ${ }^{84}$ EhFormin 1 has also been implicated in trophozoite proliferation and cytokinesis. ${ }^{87}$ (b) EhRacA nucleotide exchange is known to be accelerated by EhGEF3 in vitro. ${ }^{102}$ Constitutively active EhRacA perturbs phagocytosis and chemotaxis, as well as surface receptor capping in trophozoites. ${ }^{89}$ EhRacA.guanosine triphosphate (GTP) was also shown to bind $E$. histolytica p21-activated kinase 2 (EhPAK2), a likely effector whose kinase domain is implicated in collagen matrix invasion, cytokinesis and surface receptor capping. 90 Stimulation of EhPAK2 kinase activity by EhRacA is postulated but has not yet been established. DH, Dbl homology; GDP, guanosine diphosphate; $\mathrm{PH}$, pleckstrin homology.

cell and increased average DNA content per nucleus, ${ }^{87}$ suggesting that EhRho1/EhFormin1 signaling may be involved in actin polymerization in pseudopodia and/or trophozoite cell division (Figure 3a).

EhRhol has also been implicated in signaling downstream of lysophosphatidic acid, an agent that promotes actin polymerization and associated F-actin structures, alters concanavalin A (ConA)-induced surface receptor capping, increases migration and invasion and modulates erythrophagocytosis by E. histolytica trophozoites. ${ }^{54,88}$ Lysophosphatidic acid treatment (of the order of $10 \mu \mathrm{m}$ concentration) has been reported to promote EhRhol activation within E. histolytica, as measured by a GST (glutathione $S$-transferase)-Rhotekin Rhobinding domain pull-down assay. ${ }^{54,88}$ However, we and others have been unable to observe nucleotide-specific interaction 
between GST-Rhotekin Rho-binding domain and either epitope-tagged EhRhol expressed in cells or purified recombinant EhRhol (unpublished data), suggesting that EhRho1 binding observed in other studies ${ }^{54,88}$ may be the result of non-specific interactions, or that the employed anti-EhRhol antibody may cross-react with one or more other E. histolytica Rho family GTPases. Lysophosphatidic acid-induced EhRhol activation has also been assessed by co-immunoprecipitation with a human antigen-derived anti-Rho kinase 2 antibody; ${ }^{54}$ however no Rho kinase 2 homologs in E. histolytica have yet been described or are apparent in the genome. ${ }^{68}$

A number of Rac GTPase homologs are also expressed in E. histolytica, ${ }^{72}$ including EhRacA. Overexpression of a constitutively active $\operatorname{EhRacA}(\mathrm{G} 12 \mathrm{~V})$ in trophozoites leads to delayed cell division, as well as defects in phagocytosis of bacteria, human erythrocytes and mucin-coated beads and alterations in ConA-stimulated receptor capping. ${ }^{89}$ Furthermore, EhRacA was seen to specifically engage the p21-binding domain of the p21-activated kinase (PAK) EhPAK2, both in amebic lysates and in the context of purified recombinant proteins (Figure 3b) ${ }^{90}$ PAKs are effectors for canonical Rho family GTPases, and their serine/ threonine kinase activities and/or localizations are modulated by the binding of activated $G$ proteins (reviewed in Kumar et $\left.a . .^{91}\right)$. Trophozoites engineered to overexpress the kinase domain of EhPAK2, but not the full-length protein or the N-terminal regulatory region, exhibit defects in collagen matrix invasion, surface receptor capping and cytokinesis. ${ }^{90}$ Phenotypic overlap between $\operatorname{EhRacA}(\mathrm{G} 12 \mathrm{~V})$ and EhPAK2 kinase domain strains suggests a role for EhRacA/EhPAK2 signaling in surface receptor capping and regulation of cell division.

EhRacG has also been identified as a contributor to pathogenesis-related functions in E. histolytica. Overexpression of constitutively active $\operatorname{EhRacG}(\mathrm{G} 12 \mathrm{~V})$ in trophozoites leads to formation of a minor population of giant multinucleated cells, indicating a likely cytokinesis defect. ${ }^{92}$ Filamentous actin arrangements and surface receptor capping are also altered with EhRacG(G12V) expression, and electron microscopy observations suggest increased budding of membrane vesicles. $^{92}$ Endogenously expressed EhRacG is enriched in ConA-induced uroids, together with filamentous actin and myosin II, consistent with its regulatory role in surface receptor capping via modulation of the actin cytoskeleton. ${ }^{92}$

Activated EhRacC was recently shown to directly engage the heterotrimeric $G$ protein effector EhRGS-RhoGEF. ${ }^{63}$ Expression of constitutively active EhRacC, together with constitutively active EhG $\alpha 1$ is required to achieve EhRGSRhoGEF activation in Drosophila S2 cells, ${ }^{63}$ suggesting a convergence with heterotrimeric $G$ protein signaling (Figure 2). However, the contributions of EhRacC to cellular processes in E. histolytica remain to be directly assessed.

A number of other putative Rho family GTPase effectors have been described in E. histolytica, although without unequivocally associated G proteins. For instance, two other diaphanous-related formins with GBD-FH3 domain tandems are encoded by the E. histolytica genome in addition to the EhRho1 effector EhFormin1. ${ }^{87}$ Overexpressed EhFormin2 in trophozoites, like EhFormin1, is localized in pseudopodia and pinocytic and phagocytic vesicles, and results in mitosis and cytokinesis defects, ${ }^{87}$ suggesting some functional redundancy among diaphanous-related formins despite differences in their Rho-GTPase binding sites and, thus, likely differences in Rho activator specificities (Bosch et al. ${ }^{84}$ and unpublished data). A fourth GBD-FH3 tandem protein, the actin-binding EhNCABP166, has also been implicated as a modulator of phagocytosis, chemotactic migration and possibly proliferation in trophozoites. ${ }^{93}$ The small $G$ protein specificity of the EhNACAP166 GBD-FH3 domain tandem has been investigated; however, these binding experiments were conducted with denatured Rho GTPases, ${ }^{93}$ and intact Rho tertiary structure is required for the typical Rho/GBD-FH3 association (for example, Bosch et al. ${ }^{84}$ ). Some of the seven identified PAK family members, in addition to the EhRacA effector EhPAK2, have also been studied in E. histolytica. EhPAK (also called EhPAK1) localizes to pseudopods during amebic migration and to the uroid upon ConA-induced capping. ${ }^{94}$ The N-terminus of EhPAK1 was found to bind human Racl with typical nucleotide specificity (that is, dependent on the GTP-bound state) despite the lack of an identifiable p21-binding domain; trophozoites overexpressing the EhPAK1 kinase domain exhibit reduced migration, an increased number of membrane extensions and an increased rate of erythrocyte phagocytosis. ${ }^{95}$ EhPAK3 is also expressed in trophozoites, and both recombinant EhPAK3 purified under denaturing conditions and EhPAK3 immunoprecipitated from amebic lysates exhibit apparent kinase activity. ${ }^{96}$

Putative regulators of Rho family GTPase nucleotide cycling are also prominent in the E. histolytica genome, ${ }^{30}$ including $\sim 70$ Dbl homology (DH) domain-containing candidate RhoGEFs, $\sim 70$ encoded RhoGAP domaincontaining proteins and a single RhoGDI (EhRhoGDI; for example, Bosch et al. ${ }^{73}$ ). Although no studies of RhoGAP proteins have yet emerged, they are likely to regulate pathogenesis-related functions like their associated GTPases. Recombinant purified EhRhoGDI binds EhRhol in a nucleotide state- and isoprenylation-dependent fashion. ${ }^{73}$ As the only apparent RhoGDI, it is likely that this protein also engages other inactive Rho GTPases in E. histolytica to impair nucleotide exchange and regulate their subcellular localization.

Better studied are a number of Dbl family RhoGEFs. For example, overexpression of EhGEF1 in trophozoites decreases total cellular filamentous actin, reduces amebic migration and alters killing of mammalian cells. ${ }^{97,98}$ In vitro nucleotide exchange assays indicate that EhGEF1 likely catalyzes exchange on EhRacG and EhRhol (the latter illustrated in Figure 3a), although concentrations of GEF protein employed in these assays as well as a concentration-response analysis were not included in this report. ${ }^{98}$ Later studies have used structural homology models to predict EhGEF1 DH domain 
point mutations that impair GEF activity toward EhRhol and EhRacG, as indicated by maximal nucleotide analog fluorescence at a single time point. ${ }^{99}$ However, kinetic analysis is a preferable measure of GEF activity, as maximal fluorescence readings are subject to artifacts due to differing specific activities of recombinant proteins, non-specific binding, fluctuations in instrumentation settings, and/or 'buffer shifts' in fluorescence that vary among protein preparations. EhGEF1 small-molecule inhibitors have also been pursued, based on a docking analysis using a homology model to existing mammalian RhoGEF structures ( $\sim 50 \%$ or less sequence similarity). ${ }^{100}$ Five compounds were assessed for EhGEF1 inhibition by in vitro nucleotide exchange assays and found to be active at $\sim 50-100 \mu \mathrm{M}$ concentrations. ${ }^{100}$ However, exchange kinetics were not assessed, a typical concentrationresponse pattern was not obtained and direct binding of compounds to EhGEF1 (or potentially Rho GTPases) has not yet been demonstrated in these studies. Furthermore, the specificity of these potential pharmacological tools, for instance across other E. histolytica RhoGEFs, remains to be determined.

The Armadillo-repeat containing EhGEF2 has been implicated in erythrocyte phagocytosis, trophozoite proliferation and chemotaxis, based upon an E. histolytica strain engineered to overexpress a dominant-negative point mutant. ${ }^{101}$ Both the $\mathrm{N}$-terminal and $\mathrm{DH}$ domain regions were seen to contribute to EhGEF2 membrane localization. EhGEF2 was also suggested to activate EhRacA-D, EhRacG-H and EhCdc42 in vitro, ${ }^{101}$ although no kinetic analysis was provided in this report and the fluorescence time courses shown appear to be caused by buffer shifts upon GEF addition rather than a single exponential binding event per se. Which Rho substrates and dominant-negative mutant-impaired signals are relevant for the observed in vivo effects are currently unknown.

The DH-PH (pleckstrin homology) domain tandem of a third Dbl family RhoGEF, EhGEF3, stimulates nucleotide exchange on EhRacA and EhRhol in vitro. ${ }^{102}$ Simultaneous EhGEF3 and EhRacA overexpression in E. histolytica leads to increased migration toward fibronectin, whereas a dominant-negative EhGEF3 mutant has the opposite effect. Overexpressed EhGEF3, but not the dominant-negative point mutant, also promotes EhRacA activation in trophozoites, as assessed by a GST-EhPAK2 p21-binding domain pull-down assay, suggesting a role for EhGEF3/EhRacA signaling in chemotactic migration (Figure 3b). ${ }^{102}$ EhGEF3 and EhRacA co-localize in caps induced by ConA treatment, suggesting a possible additional role in surface receptor capping. ${ }^{102}$

Members of a family of 11 RhoGEFs in E. histolytica each contain a FYVE domain, known to associate with inositol phospholipids and to decorate early phagosomes in trophozoites. $^{103}$ A GFP-tagged mammalian FYVE domain overexpressed in trophozoites was observed to translocate to phagocytic cups and phagosomes during host cell phagocytosis. ${ }^{104}$ One overexpressed FYVE domain-containing RhoGEF, EhFP4, is also recruited to phagocytosis-related structures, ${ }^{104}$ and overexpression of the isolated FYVE domain from EhFP4 impairs trophozoite phagocytosis. Interaction of EhFP4 or its DH-PH domains with recombinant $E$. histolytica Rho family GTPases has been assessed with pull-down assays. EhFP4 was seen to interact with EhRacC, EhRacD, and two unnamed G proteins, although nucleotide state selectivity was not assessed in this study, and the authors reported inability to detect nucleotide exchange activity. ${ }^{104}$ Thus, it remains to be established whether these FYVE domain-containing RhoGEFs exhibit GEF activity and whether there is any functional interplay between their FYVE and DH-PH domains.

\section{Rab family GTPases}

The E. histolytica genome encodes a remarkable 91 Rab family $\mathrm{G}$ proteins, many of which are not clear homologs of mammalian Rabs, suggesting an unusually high degree of complexity underlying vesicular trafficking regulation in trophozoites. ${ }^{74,105,106}$ Endosomes isolated from E. histolytica by magnetic fractionation are associated with virulenceassociated cysteine protease activity, as well as enrichment of Rab GTPases, such as EhRab11 and potentially a Rab7 homolog. ${ }^{107}$ The importance of phagocytosis and pinocytosis to nutrient uptake by trophozoites, the secretion of virulence factors like amebapores and cysteine proteases, as well as the critical role of membrane-associated proteins like the Gal/GalNAc lectin to pathogenic behaviors, all support the hypothesis that Rab-regulated vesicular trafficking is important for E. histolytica biology and pathogenesis. ${ }^{14,23,24}$

EhRabA is localized to vesicles at steady state, but moves to the leading edge in motile cells and the membrane opposite ConA-induced caps, as well as to membrane extensions upon $\mathrm{N}$-formyl peptide-induced polarization. ${ }^{108}$ Expression of a dominant-negative EhRabA mutant in trophozoites produces changes in cell morphology and polarization, impairs motility and reduces host cell attachment and killing but has no observable effect on pinocytosis or erythrophagocytosis. ${ }^{109}$ Conversely, overexpression of constitutively active EhRabA perturbs erythrophagocytosis and leads to formation of large tubular organelles apparently derived from the endoplasmic reticulum (ER). ${ }^{110}$ Two subunits of the Gal/Gal-NAc lectin and a cysteine protease are mislocalized to these EhRabA-induced organelles, and similar effects are seen with brefeldin-A treatment, suggesting that EhRabA regulates trafficking between the ER and Golgi apparatus in E. histolytica. ${ }^{110}$

EhRabB is one of the first identified and most frequently studied Rab family GTPases in E. histolytica. Initial immunofluorescence studies localized endogenous EhRabB to cytoplasmic vesicles and noted its translocation to the plasma membrane and phagocytic cups during erythrophagocytosis. ${ }^{111,112}$ Poor phagocytosis in a mutant E. histolytica strain was seen to correlate with increased expression of EhRabB, as well as substantial sequence differences between this mutant EhRabB and wild-type EhRabB, providing further evidence for its involvement in phagocytosis, ${ }^{113}$ although a causal association was not established. EhRabB was also observed to 
be enriched at phagosomes in a proteomics study. ${ }^{114}$ Overexpression of wild-type EhRabB in trophozoites leads to a small diminution of phagocytosis, while expression of a dominant-negative mutant (N118I) leads to decreases in both phagocytosis and cell monolayer destruction. ${ }^{115}$ Of particular interest, EhRabB(N118I)-expressing trophozoites do not form liver abscesses in a hamster model, while vector-transfected and wild-type EhRabB-expressing amebae do form such abscesses, establishing EhRabB signaling as likely important for pathogenesis. ${ }^{115}$ EhRabB was reported to interact with the transmembrane protein EhGPCR-1 by yeast two-hybrid, although binding data were not shown in this study. ${ }^{116}$ Despite its initial naming as a GPCR, further sequence analysis has indicated that EhGPCR-1 is more likely a Wnt-binding factor rather than a ligand-activated heterotrimeric $G$ protein GEF per se. ${ }^{61}$

E. histolytica also expresses Rab5- and Rab7-related G proteins. ${ }^{117,118}$ Overexpressed EhRab5 and EhRab7A both localize to independent vesicular structures at steady state, but exposure of these overexpression-modified trophozoites to erythrocytes is seen to cause convergence of the two Rabs at a large 'pre-phagosomal vacuole', distinct from actual phagosomes. ${ }^{119}$ Electron microscopic studies have identified small amebapore-containing vesicles in the pre-phagosomal vacuole, suggesting a role for EhRab5 and/or EhRab7A in delivering cytotoxic amebapores to phagosomes. ${ }^{119}$ Consistent with this hypothesis, overexpression of wild-type EhRab5 enhances phagocytosis kinetics and amebapore transport, while expression of either constitutively active or dominantnegative EhRab5 mutants impairs pre-phagosomal vacuole formation and phagocytosis. ${ }^{119}$ EhRab7 also co-localizes with early endosomes, ${ }^{117}$ and overexpression of EhRab7A in trophozoites reveals its subcellular localization to lysosomes and an increased acidic cellular compartment as well as decreased cellular cysteine protease activity. ${ }^{120}$ A retromerlike complex of $E$. histolytica proteins is seen to engage recombinant EhRab7A in a nucleotide-dependent fashion, primarily through the C-terminus of EhVps26, leading to the hypothesis that EhRab7A may contribute to retrograde transport from vacuoles and phagosomes to the Golgi apparatus. ${ }^{120}$ An E. histolytica homolog of Rab8 has also been cloned, but no cellular functions have yet been established for this $G$ protein other than its vesicular localization. ${ }^{121,122}$

EhRab11 exhibits a punctate distribution in trophozoites and moves to the cell periphery upon iron and serum starvation of trophozoites, ${ }^{123}$ in contrast to EhRab7 and EhRabA. Iron and serum starvation is associated with altered cytokinesis and increased detergent-resistant cells, but whether EhRab11 contributes to these phenotypes is unknown. ${ }^{123} \mathrm{~A}$ related isoform, EhRab11B, also exhibits a vesicular distribution, and overexpression of EhRab11B in trophozoites leads to an increase in both intracellular and secreted cysteine proteases. ${ }^{124}$ Amebae overexpressing EhRab11B exhibit slightly increased exocytosis of a fluidphase marker and more efficiently kill mammalian cells, an effect reversed by treatment with the cysteine protease inhibitor E64. ${ }^{124}$ These findings suggest that the E. histolytica Rab11 isoforms have non-redundant functions.

No studies of Rab GTPase nucleotide cycle regulators in $E$. histolytica have yet emerged. The E. histolytica genome encodes for $\sim 20$ proteins with DENN (after 'differentially expressed in neoplastic vs normal' cells) domains, ${ }^{\overline{6}}$ known in mammals to serve as Rab GEFs, along with other structurally unrelated proteins. ${ }^{125}$ Also present are $\sim 50$ Rab-GAP/TBC (Tre-2, Bub2, and Cdc16) domain-containing proteins and two putative Rab GDIs. ${ }^{68}$ Examination of these likely Rab regulators may shed further light on signaling mechanisms contributing to E. histolytica pathogenicity, especially in the context of vesicular trafficking mechanics.

\section{CONCLUSION AND PERSPECTIVE}

A number of $G$ proteins have been implicated in key pathogenic processes of E. histolytica, particularly heterotrimeric $G$ proteins, a number of cytoskeleton-associated Rho GTPases, and also Rab GTPases primarily involved in vesicular trafficking. Exploitation of known signaling pathways for pharmacological manipulation is attractive, both in the development of tools for interrogating the specific functions of $G$ protein signaling in E. histolytica and as a potential approach to the development of anti-amebiasis therapeutics. A first step has been taken in developing small molecule inhibitors for EhGEF $1,{ }^{100}$ and other E. histolytica RhoGEFs may be targetable given some previous success in inhibiting mammalian Rho GTPase activation (for example, Shutes et al. ${ }^{126}$ and Evelyn et al. ${ }^{127}$ ). Some Ras and Rho GTPase effectors, particularly kinases like the PAKs and members of the mitogen-activated protein kinase cascade, have also proven tractable as pharmacological targets in humans (for example, Rusconi et al. ${ }^{128}$ and Zhao and Manser ${ }^{129}$ ). However, the importance of Ras effectors and downstream kinases in E. histolytica pathogenesis has not yet been explored. Particularly promising for pharmacological development is the recently described heterotrimeric $G$ protein signaling within E. histolytica, ${ }^{36,61}$ although identification of a bona fide GPCR and ligand pair in E. histolytica remains a barrier at this time.

Aside from pharmacological goals, much remains to be discovered regarding the modulation of Ras superfamily GTPase functions in E. histolytica, particularly regarding nucleotide cycle regulators (GAPs, GEFs and GDIs). Also unclear is the interplay among the well-populated small $G$ protein families in E. histolytica, such as the $>20$-member Rho family and the 91-member Rab family. Are many of these GTPases redundant in function and regulation? How is GTPase specificity for effectors and nucleotide cycle regulators achieved, given such large numbers of simultaneously expressed G proteins in a single cell? Further study of both heterotrimeric and small G proteins in E. histolytica will likely add to our understanding of parasite biology and pathogenicity, as well as signaling in other organisms. 
1 Ravdin JI. Amoebiasis. Imperial College Press: London, 2000.

2 WHO. WHO/PAHO/UNESCO report. A consultation with experts on amoebiasis. Mexico City, Mexico 28-29 January, 1997. Epidemiol Bull 1997; 18: 13-14.

3 Haque R, Huston CD, Hughes M, Houpt E, Petri WA Jr. Amebiasis. N Engl J Med 2003; 348: 1565-1573.

4 Korpe PS, Stott BR, Nazib F, Kabir M, Haque R, Herbein JF et al. Evaluation of a rapid point-of-care fecal antigen detection test for Entamoeba histolytica. Am J Trop Med Hyg 2012; 86: 980-981.

5 Haque R, Kabir M, Noor Z, Rahman SM, Mondal D, Alam F et al. Diagnosis of amebic liver abscess and amebic colitis by detection of Entamoeba histolytica DNA in blood, urine, and saliva by a real-time PCR assay. J Clin Microbiol 2010; 48: 2798-2801.

6 Haque R, Mondal D, Duggal P, Kabir M, Roy S, Farr BM et al. Entamoeba histolytica infection in children and protection from subsequent amebiasis. Infect Immun 2006; 74: 904-909.

7 Anuar TS, Al-Mekhlafi HM, Abdul Ghani MK, Abu Bakar E, Azreen SN, Salleh FM et al. Molecular epidemiology of amoebiasis in Malaysia: highlighting the different risk factors of Entamoeba histolytica and Entamoeba dispar infections among Orang Asli communities. Int J Parasitol 2012; 42: 1165-1175.

8 Ramos F, Moran P, Gonzalez E, Garcia G, Ramiro M, Gomez A et al. High prevalence rate of Entamoeba histolytica asymptomatic infection in a rural Mexican community. Am J Trop Med Hyg 2005; 73: 87-91.

9 Yang B, Chen Y, Wu L, Xu L, Tachibana H, Cheng X. Seroprevalence of Entamoeba histolytica infection in China. Am J Trop Med Hyg 2012; 87: 97-103.

10 Verkerke HP, Petri WA Jr, Marie CS. The dynamic interdependence of amebiasis, innate immunity, and undernutrition. Semin Immunopathol 2012; 34: 771-785.

11 Hung CC, Chang SY, Ji DD. Entamoeba histolytica infection in men who have sex with men. Lancet Infect Dis 2012; 12: 729-736.

12 Nozaki T, Kobayashi S, Takeuchi T, Haghighi A. Diversity of clinical isolates of Entamoeba histolytica in Japan. Arch Med Res 2006; 37: 277-279.

13 Barwick RS, Uzicanin A, Lareau S, Malakmadze N, Imnadze P, Iosava M et al. Outbreak of amebiasis in Tbilisi, Republic of Georgia, 1998. Am J Trop Med Hyg 2002; 67: 623-631.

14 Christy NC, Petri WA Jr. Mechanisms of adherence, cytotoxicity and phagocytosis modulate the pathogenesis of Entamoeba histolytica. Future Microbiol 2011; 6: 1501-1519.

15 Aikat BK, Bhusnurmath SR, Pal AK, Chhuttani PN, Datta DV. The pathology and pathogenesis of fatal hepatic amoebiasis-a study based on 79 autopsy cases. Trans R Soc Trop Med Hyg 1979; 73: 188-192.

16 Knobloch J, Mannweiler E. Development and persistence of antibodies to Entamoeba histolytica in patients with amebic liver abscess. Analysis of 216 cases. Am J Trop Med Hyg 1983; 32: 727-732.

17 Lofmark S, Edlund C, Nord CE. Metronidazole is still the drug of choice for treatment of anaerobic infections. Clin Infect Dis 2010; 50 (Suppl 1): S16-S23.

18 Bansal D, Malla N, Mahajan RC. Drug resistance in amoebiasis. Indian J Med Res 2006; 123: 115-118.

19 Debnath A, Parsonage D, Andrade RM, He C, Cobo ER, Hirata K et al. A high-throughput drug screen for Entamoeba histolytica identifies a new lead and target. Nat Med 2012; 18: 956-960.

20 Wani MY, Bhat AR, Azam A, Lee DH, Choi I, Athar F. Synthesis and in vitro evaluation of novel tetrazole embedded 1,3,5-trisubstituted pyrazoline derivatives as Entamoeba histolytica growth inhibitors. Eur J Med Chem 2012; 54: 845-854.

21 Mushtaque M, Avecilla F, Azam A. Synthesis, characterization and structure optimization of a series of thiazolidinone derivatives as Entamoeba histolytica inhibitors. Eur J Med Chem 2012; 55: 439-448.

22 Abd Alla MD, Wolf R, White GL, Kosanke SD, Cary D, Verweij JJ et al. Efficacy of a Gal-lectin subunit vaccine against experimental Entamoeba histolytica infection and colitis in baboons (Papio sp.). Vaccine 2012; 30: 3068-3075.

23 Petri WA Jr, Haque R, Mann BJ. The bittersweet interface of parasite and host: lectin-carbohydrate interactions during human invasion by the parasite Entamoeba histolytica. Annu Rev Microbiol 2002; 56: 39-64.

24 Leippe M, Herbst R. Ancient weapons for attack and defense: the poreforming polypeptides of pathogenic enteric and free-living amoeboid protozoa. J Eukaryot Microbiol 2004; 51: 516-521.
25 Que X, Reed SL. Cysteine proteinases and the pathogenesis of amebiasis. Clin Microbiol Rev 2000; 13: 196-206.

26 Voigt $H$, Guillen N. New insights into the role of the cytoskeleton in phagocytosis of Entamoeba histolytica. Cell Microbiol 1999; 1: 195-203.

27 Tavares P, Sansonetti P, Guillen N. The interplay between receptor capping and cytoskeleton remodeling in Entamoeba histolytica. Arch Med Res 2000; 31: S140-S142.

28 Meza I, Talamas-Rohana P, Vargas MA. The cytoskeleton of Entamoeba histolytica: structure, function, and regulation by signaling pathways. Arch Med Res 2006; 37: 234-243.

29 Labruyere E, Guillen N. Host tissue invasion by Entamoeba histolytica is powered by motility and phagocytosis. Arch Med Res 2006; 37: 253-258.

30 Loftus B, Anderson I, Davies R, Alsmark UC, Samuelson J, Amedeo $\mathrm{P}$ et al. The genome of the protist parasite Entamoeba histolytica. Nature 2005; 433: 865-868.

31 Gilchrist CA, Houpt E, Trapaidze N, Fei Z, Crasta O, Asgharpour A et al. Impact of intestinal colonization and invasion on the Entamoeba histolytica transcriptome. Mol Biochem Parasitol 2006; 147: 163-176.

32 Anamika K, Bhattacharya A, Srinivasan N. Analysis of the protein kinome of Entamoeba histolytica. Proteins 2008; 71: 995-1006.

33 Wennerberg K, Rossman KL, Der CJ. The Ras superfamily at a glance. J Cell Sci 2005; 118: 843-846.

34 Oldham WM, Hamm HE. Heterotrimeric G protein activation by G-protein-coupled receptors. Nat Rev Mol Cell Biol 2008; 9: 60-71.

35 Wennerberg K, Der CJ. Rho-family GTPases: it's not only Rac and Rho (and I like it). J Cell Sci 2004; 117: 1301-1312.

36 Gilchrist A. Modulating G-protein-coupled receptors: from traditional pharmacology to allosterics. Trends Pharmacol Sci 2007; 28: 431-437.

37 Rask-Andersen M, Almen MS, Schioth HB. Trends in the exploitation of novel drug targets. Nat Rev Drug Discov 2011; 10: 579-590.

38 Rasmussen SG, DeVree BT, Zou Y, Kruse AC, Chung KY, Kobilka TS et al. Crystal structure of the beta2 adrenergic receptor-Gs protein complex. Nature 2011; 477: 549-555.

39 Coleman DE, Berghuis AM, Lee E, Linder ME, Gilman AG, Sprang SR. Structures of active conformations of Gi alpha 1 and the mechanism of GTP hydrolysis. Science 1994; 265: 1405-1412.

40 Aittaleb M, Boguth CA, Tesmer JJ. Structure and function of heterotrimeric $\mathrm{G}$ protein-regulated Rho guanine nucleotide exchange factors. Mol Pharmacol 2010; 77: 111-125.

41 Kimple AJ, Bosch DE, Giguere PM, Siderovski DP. Regulators of G-protein signaling and their Galpha substrates: promises and challenges in their use as drug discovery targets. Pharmacol Rev 2011; 63: 728-749.

42 Tesmer JJ, Berman DM, Gilman AG, Sprang SR. Structure of RGS4 bound to AIF4-activated G(i alpha1): stabilization of the transition state for GTP hydrolysis. Cell 1997; 89: 251-261.

43 Waldo GL, Ricks TK, Hicks SN, Cheever ML, Kawano T, Tsuboi K et al. Kinetic scaffolding mediated by a phospholipase C-beta and Gq signaling complex. Science 2010; 330: 974-980.

44 Kimple RJ, Kimple ME, Betts L, Sondek J, Siderovski DP. Structural determinants for GoLoco-induced inhibition of nucleotide release by Galpha subunits. Nature 2002; 416: 878-881.

45 Worthylake DK, Rossman KL, Sondek J. Crystal structure of Rac1 in complex with the guanine nucleotide exchange region of Tiam1. Nature 2000; 408: 682-688.

46 Scheffzek K, Ahmadian MR, Kabsch W, Wiesmuller L, Lautwein A, Schmitz $\mathrm{F}$ et al. The Ras-RasGAP complex: structural basis for GTPase activation and its loss in oncogenic Ras mutants. Science 1997; 277: 333-338.

47 Gavriljuk K, Gazdag EM, Itzen A, Kotting C, Goody RS, Gerwert K. Catalytic mechanism of a mammalian Rab\{middle dot\}RabGAP complex in atomic detail. Proc Natl Acad Sci USA 2012; 109: 21348-21353.

48 Garcia-Mata R, Boulter E, Burridge K. The 'invisible hand': regulation of RHO GTPases by RHOGDIs. Nat Rev Mol Cell Biol 2011; 12: 493-504.

49 Scheffzek K, Stephan I, Jensen ON, Illenberger D, Gierschik P. The RacRhoGDI complex and the structural basis for the regulation of Rho proteins by RhoGDI. Nat Struct Biol 2000; 7: 122-126.

50 Khan MA, Sen PC. Demonstration of histamine receptors on the surface of Entamoeba histolytica. Indian J Exp Biol 1989; 27: 96-97.

51 Khan MA, Sen PC. Effect of histamine and serotonin on phagocytic activity of Entamoeba histolytica. Indian J Exp Biol 1988; 26: 730-731. 
52 Khan MA, Sen PC. Modulation of pathogenicity of Entamoeba histolytica by histamine. Indian J Med Res 1988; 88: 225-227.

53 Khan MA, Sen PC, Mishra B. Enhancement of virulence of Entamoeba histolytica by histamine in vitro. Indian J Exp Biol 1990; 28: 376-377.

54 Rios A, Hernandez-Ramirez VI, Moguel M, Bahena AIZ, Rosales-Encina $\mathrm{JL}$, Vargas MA et al. Participation of Rho, ROCK-2, and GAP activities during actin microfilament rearrangements in Entamoeba histolytica induced by fibronectin signaling. Cell Biol Int 2008; 32: 984-1000.

55 Manning-Cela R, Pina A, Meza I. cAMP levels and up-regulation of actin mRNA in Entamoeba histolytica. Arch Med Res 1997; 28 (Spec No): 134-135.

56 Carbajal ME, Manning-Cela R, Pina A, Franco E, Meza I. Fibronectininduced intracellular calcium rise in Entamoeba histolytica trophozoites: effect on adhesion and the actin cytoskeleton. Exp Parasitol 1996; 82: 11-20.

57 Siderovski DP, Kimple AJ, Willard FS. Large G-proteins. In: Begley TP (ed.). Wiley Encyclopedia of Chemical Biology. John Wiley \& Sons, Inc., 2008, pp 1-9.

58 Soid-Raggi LG, Torres-Marquez ME, Meza I. Entamoeba histolytica: identification of functional $\mathrm{Gs}$ and $\mathrm{Gi}$ proteins as possible signal transduction elements in the interaction of trophozoites with fibronectin. Exp Parasitol 1998; 90: 262-269.

59 Coppi A, Merali S, Eichinger D. The enteric parasite Entamoeba uses an autocrine catecholamine system during differentiation into the infectious cyst stage. J Biol Chem 2002; 277: 8083-8090.

60 Frederick J, Eichinger D. Entamoeba invadens contains the components of a classical adrenergic signaling system. Mol Biochem Parasitol 2004; 137: 339-343.

61 Bosch DE, Kimple AJ, Muller RE, Giguere PM, Machius M, Willard FS et al. Heterotrimeric G-protein signaling is critical to pathogenic processes in Entamoeba histolytica. PLoS Pathog 2012; 8: e1003040.

62 Anantharaman V, Abhiman S, de Souza RF, Aravind L. Comparative genomics uncovers novel structural and functional features of the heterotrimeric GTPase signaling system. Gene 2011; 475: 63-78.

63 Bosch DE, Kimple AJ, Manning AJ, Muller RE, Willard FS, Machius M et al. Structural determinants of RGS-RhoGEF signaling critical to Entamoeba histolytica pathogenesis. Structure 2013; 21: 65-75.

64 Garcia-Marcos M, Ghosh P, Farquhar MG. GIV is a nonreceptor GEF for G alpha i with a unique motif that regulates Akt signaling. Proc Natl Acad Sci USA 2009; 106: 3178-3183.

65 Wittinghofer A, Vetter IR. Structure-function relationships of the G domain, a canonical switch motif. Annu Rev Biochem 2011; 80: 943-971.

66 Johnston CA, Willard MD, Kimple AJ, Siderovski DP, Willard FS. A sweet cycle for Arabidopsis G-proteins: recent discoveries and controversies in plant G-protein signal transduction. Plant Signal Behav 2008; 3: 1067-1076.

67 Slessareva JE, Dohlman HG. G protein signaling in yeast: new components, new connections, new compartments. Science 2006; 314: 1412-1413.

68 Aurrecoechea C, Barreto A, Brestelli J, Brunk BP, Caler EV, Fischer S et al. AmoebaDB and MicrosporidiaDB: functional genomic resources for Amoebozoa and Microsporidia species. Nucleic Acids Res 2011; 39: D612-D619.

69 Shen PS, Lohia A, Samuelson J. Molecular cloning of ras and rap genes from Entamoeba histolytica. Mol Biochem Parasitol 1994; 64: 111-120.

70 Kumagai M, Makioka A, Takeuchi T, Nozaki T. Molecular cloning and characterization of a protein farnesyltransferase from the enteric protozoan parasite Entamoeba histolytica. J Biol Chem 2004; 279: 2316-2323.

71 Lohia A, Samuelson J. Molecular cloning of a rho family gene of Entamoeba histolytica. Mol Biochem Parasitol 1993; 58: 177-180.

72 Lohia A, Samuelson J. Heterogeneity of Entamoeba histolytica rac genes encoding p21rac homologues. Gene 1996; 173: 205-208.

73 Bosch DE, Wittchen ES, Qiu C, Burridge K, Siderovski DP. Unique structural and nucleotide exchange features of the Rhol GTPase of Entamoeba histolytica. J Biol Chem 2011; 286: 39236-39246.

74 Nakada-Tsukui K, Saito-Nakano Y, Husain A, Nozaki T. Conservation and function of Rab small GTPases in Entamoeba: annotation of $E$. invadens Rab and its use for the understanding of Entamoeba biology. Exp Parasitol 2010; 126: 337-347.

75 Shen PS, Sanford JC, Samuelson J. Entamoeba histolytica: isoprenylation of p21ras and p21rap in vitro. Exp Parasitol 1996; 82: 65-68.

76 Baines AT, Xu D, Der CJ. Inhibition of Ras for cancer treatment: the search continues. Future Med Chem 2011; 3: 1787-1808.
77 Marion S, Laurent C, Guillen N. Signalization and cytoskeleton activity through myosin IB during the early steps of phagocytosis in Entamoeba histolytica: a proteomic approach. Cell Microbiol 2005; 7: 1504-1518.

78 Maugis B, Brugues J, Nassoy P, Guillen N, Sens P, Amblard F. Dynamic instability of the intracellular pressure drives bleb-based motility. J Cell Sci 2010; 123: 3884-3892.

79 Meza I. Extracellular matrix-induced signaling in Entamoeba histolytica: its role in invasiveness. Parasitol Today 2000; 16: 23-28.

80 Zaki M, Andrew N, Insall RH. Entamoeba histolytica cell movement: a central role for self-generated chemokines and chemorepellents. Proc Natl Acad Sci USA 2006; 103: 18751-18756.

81 Godbold GD, Mann BJ. Cell killing by the human parasite Entamoeba histolytica is inhibited by the Rho-inactivating C3 exoenzyme. Mol Biochem Parasitol 2000; 108: 147-151.

82 Godbold GD, Corbett KD, Mann BJA. Rho-like small GTPase of Entamoeba histolytica contains an unusual amino acid residue in a conserved GDP-stabilization region and is not a substrate for C3 exoenzyme. Exp Parasitol 2002; 101: 107-110.

83 Majumder S, Schmidt G, Lohia A, Aktories K. EhRho1, a RhoA-like GTPase of Entamoeba histolytica, is modified by clostridial glucosylating cytotoxins. Appl Environ Microbiol 2006; 72: 7842-7848.

84 Bosch DE, Yang B, Siderovski DP. Entamoeba histolytica Rhol regulates actin polymerization through a divergent, diaphanous-related formin. Biochemistry 2012; 51: 8791-8801.

85 Chesarone MA, DuPage AG, Goode BL. Unleashing formins to remodel the actin and microtubule cytoskeletons. Nat Rev Mol Cell Biol 2010; 11: $62-74$.

86 Ganguly A, Lohia A. The diaphanous protein from Entamoeba histolytica controls cell motility and cytokinesis. Arch Med Res 2000; 31: S137-S139.

87 Majumder S, Lohia A. Entamoeba histolytica encodes unique formins, a subset of which regulates DNA content and cell division. Infect Immun 2008; 76: 2368-2378.

88 Franco-Barraza J, Zamudio-Meza H, Franco E, MdC Dominguez-Robles, Villegas-Sepulveda N, Meza I. Rho sgnaling in Entamoeba histolytica modulates actomyosin-dependent activities stimulated during invasive behavior. Cell Motil Cytoskeleton 2006: 117-131.

89 Ghosh SK, Samuelson J. Involvement of p21racA, phosphoinositide 3-kinase, and vacuolar ATPase in phagocytosis of bacteria and erythrocytes by Entamoeba histolytica: suggestive evidence for coincidental evolution of amebic invasiveness. Infect Immun 1997; 65: 4243-4249.

90 Arias-Romero LE, de Jesus Almaraz-Barrera M, Diaz-Valencia JD, Rojo-Dominguez A, Hernandez-Rivas R, Vargas M. EhPAK2, a novel p21-activated kinase, is required for collagen invasion and capping in Entamoeba histolytica. Mol Biochem Parasitol 2006; 149: 17-26.

91 Kumar A, Molli PR, Pakala SB, Bui Nguyen TM, Rayala SK, Kumar R. PAK thread from amoeba to mammals. J Cell Biochem 2009; 107: 579-585.

92 Guillen N, Boquet P, Sansonetti P. The small GTP-binding protein RacG regulates uroid formation in the protozoan parasite Entamoeba histolytica. J Cell Sci 1998; 111(Pt 12): 1729-1739.

93 Campos-Parra AD, Hernandez-Cuevas NA, Hernandez-Rivas R, Vargas M. EhNCABP166: a nucleocytoplasmic actin-binding protein from Entamoeba histolytica. Mol Biochem Parasitol 2010; 172: 19-30.

94 Labruyere E, Galy V, Sansonetti P, Guillen N. Distribution of a potential p21-activated serine/threonine kinase (PAK) in Entamoeba histolytica. Arch Med Res 2000; 31: S128-S130.

95 Labruyere E, Zimmer C, Galy V, Olivo-Marin J-C, Guillen N. EhPAK, a member of the p21-activated kinase family, is involved in the control of Entamoeba histolytica migration and phagocytosis. J Cell Sci 2003; 116: 61-71.

96 Dutta S, Sardar A, Ray D, Raha S. Molecular and functional characterization of EhPAK3, a p21 activated kinase from Entamoeba histolytica. Gene 2007; 402: 57-67.

97 Santiago-Resendiz M, Vargas-Mejia M. The tail domain of Entamoeba histolytica GEF: a guanine nucleotide exchange factor. Arch Med Res 2000; 31: S301-S302.

98 Aguilar-Rojas A, Almaraz-Barrera Mde J, Krzeminski M, Robles-Flores M, Hernandez-Rivas R, Guillen N et al. Entamoeba histolytica: inhibition of cellular functions by overexpression of EhGEF1, a novel Rho/Rac guanine nucleotide exchange factor. Exp Parasitol 2005; 109: 150-162.

99 Hernandez-Cuevas NA, Campos-Parra AD, Almaraz-Barrera MdJ, AguilarRojas A, Rosa CHG-dl, Sosa-Peinado A et al. Entamoeba histolytica EhGEF1 structure and mutational analysis: new specific residues critical for function. Mol Biochem Parasitol 2009; 164: 118-125. 
100 Hernandez-Cuevas NA, Hernandez-Rivas R, Sosa-Peinado A, Rojo-Dominguez A, Vargas M. Identification and evaluation of inhibitors of the EhGEF1 protein from Entamoeba histolytica. J Mol Recognit 2011; 24: 935-944.

101 Gonzalez De la Rosa CH, Arias-Romero LE, de Jesus Almaraz-Barrera M, Hernandez-Rivas R, Sosa-Peinado A, Rojo-Dominguez A et al. EhGEF2, a Dbl-RhoGEF from Entamoeba histolytica has atypical biochemical properties and participates in essential cellular processes. Mol Biochem Parasitol 2007; 151: 70-80.

102 Arias-Romero LE, Rosa CHGdl, Almaraz-Barrera MdJ, Diaz-Valencia JD, Sosa-Peinado A, Vargas M. EhGEF3, a novel Dbl family member, regulates EhRacA activation during chemotaxis and capping in Entamoeba histolytica. Cell Motil Cytoskeleton 2007; 64: 390-404.

103 Powell RR, Welter BH, Hwu R, Bowersox B, Attaway C, Temesvari LA. Entamoeba histolytica: FYVE-finger domains, phosphatidylinositol 3phosphate biosensors, associate with phagosomes but not fluid filled endosomes. Exp Parasitol 2006; 112: 221-231.

104 Nakada-Tsukui K, Okada H, Mitra BN, Nozaki T. Phosphatidylinositolphosphates mediate cytoskeletal reorganization during phagocytosis via a unique modular protein consisting of RhoGEF/DH and FYVE domains in the parasitic protozoon Entamoeba histolytica. Cell Microbiol 2009; 11: 1471-1491.

105 Saito-Nakano Y, Nakazawa M, Shigeta Y, Takeuchi T, Nozaki T. Identification and characterization of genes encoding novel Rab proteins from Entamoeba histolytica. Mol Biochem Parasitol 2001; 116: 219-222.

106 Saito-Nakano Y, Loftus BJ, Hall N, Nozaki T. The diversity of Rab GTPases in Entamoeba histolytica. Exp Parasitol 2005; 110: 244-252.

107 Temesvari LA, Harris EN, Stanley SL Jr, Cardelli JA. Early and late endosomal compartments of Entamoeba histolytica are enriched in cysteine proteases, acid phosphatase and several Ras-related Rab GTPases. Mol Biochem Parasitol 1999; 103: 225-241.

108 Welter BH, Temesvari LA. A unique Rab GTPase, EhRabA, of Entamoeba histolytica, localizes to the leading edge of motile cells. Mol Biochem Parasitol 2004; 135: 185-195.

109 Welter BH, Powell RR, Leo M, Smith CM, Temesvari LA. A unique Rab GTPase, EhRabA, is involved in motility and polarization of Entamoeba histolytica cells. Mol Biochem Parasitol 2005; 140: 161-173.

110 Welter BH, Temesvari LA. Overexpression of a mutant form of EhRabA, a unique Rab GTPase of Entamoeba histolytica, alters endoplasmic reticulum morphology and localization of the Gal/GalNAc adherence lectin. Eukaryot Cell 2009; 8: 1014-1026.

111 Rodriguez MA, Garcia-Perez RM, Garcia-Rivera G, Lopez-Reyes I, Mendoza L, Ortiz-Navarrete $V$ et al. An Entamoeba histolytica rab-like encoding gene and protein: function and cellular location. Mol Biochem Parasitol 2000; 108: 199-206.

112 Rodriguez MA, Orozco E. Characterization of the EhRabB recombinant protein of Entamoeba histolytica. Arch Med Res 2000; 31: S171-S172.

113 Guzman-Medrano R, Castillo-Juarez BA, Garcia-Perez RM, Salas-Casas A, Orozco E, Rodriguez MA. Entamoeba histolytica: alterations in EhRabB protein in a phagocytosis deficient mutant correlate with the Entamoeba dispar RabB sequence. Exp Parasitol 2005; 110: 259-264.

114 Marion S, Guillen N. Genomic and proteomic approaches highlight phagocytosis of living and apoptotic human cells by the parasite Entamoeba histolytica. Int J Parasitol 2006; 36: 131-139.

115 Juarez-Hernandez LJ, Garcia-Perez RM, Salas-Casas A, Garcia-Rivera G, Orozco E, Rodriguez MA. Entamoeba histolytica: the over expression of a mutated EhRabB protein produces a decrease of in vitro and in vivo virulence. Exp Parasitol 2012; 133: 339-345.

116 Picazarri K, Luna-Arias JP, Carrillo E, Orozco E, Rodriguez MA. Entamoeba histolytica: identification of EhGPCR-1, a novel putative G protein-coupled receptor that binds to EhRabB. Exp Parasitol 2005; 110: 253-258.

117 Welter BH, Laughlin RC, Temesvari LA. Characterization of a Rab7-like GTPase, EhRab7: a marker for the early stages of endocytosis in Entamoeba histolytica. Mol Biochem Parasitol 2002; 121: 254-264.

118 Saito-Nakano Y, Yasuda T, Shigeta Y, Nakazawa M, Takeuchi T, Nozaki T. Identification and characterizaton of a Rab5 homologue in Entamoeba histolytica. Arch Med Res 2000; 31: S155-S156.

119 Saito-Nakano Y, Yasuda T, Nakada-Tsukui K, Leippe M, Nozaki T. Rab5associated vacuoles play a unique role in phagocytosis of the enteric protozoan parasite Entamoeba histolytica. J Biol Chem 2004; 279: 49497-49507.

120 Nakada-Tsukui K, Saito-Nakano Y, Ali V, Nozaki T. A retromerlike complex is a novel Rab7 effector that is involved in the transport of the virulence factor cysteine protease in the enteric protozoan parasite Entamoeba histolytica. Mol Biol Cell 2005; 16: 5294-5303.

121 Juarez P, Sanchez-Lopez R, Ramos MA, Stock RP, Alagon A. Rab8 as a molecular model of vesicular trafficking to investigate the latter steps of the secretory pathway in Entamoeba histolytica. Arch Med Res 2000; 31. S157-S159.

122 Juarez P, Sanchez-Lopez R, Stock RP, Olvera A, Ramos MA, Alagon A. Characterization of the Ehrab8 gene, a marker of the late stages of the secretory pathway of Entamoeba histolytica. Mol Biochem Parasitol 2001; 116: 223-228.

123 McGugan GC Jr, Temesvari LA. Characterization of a Rab11-like GTPase, EhRab11, of Entamoeba histolytica. Mol Biochem Parasitol 2003; 129: 137-146.

124 Mitra BN, Saito-Nakano Y, Nakada-Tsukui K, Sato D, Nozaki T. Rab11B small GTPase regulates secretion of cysteine proteases in the enteric protozoan parasite Entamoeba histolytica. Cell Microbiol 2007; 9: 2112-2125.

125 Barr F, Lambright DG. Rab GEFs and GAPs. Curr Opin Cell Biol 2010; 22: 461-470.

126 Shutes A, Onesto C, Picard V, Leblond B, Schweighoffer F, Der CJ. Specificity and mechanism of action of EHT 1864, a novel small molecule inhibitor of Rac family small GTPases. J Biol Chem 2007; 282: 35666-35678.

127 Evelyn CR, Ferng T, Rojas RJ, Larsen MJ, Sondek J, Neubig RR. Highthroughput screening for small-molecule inhibitors of LARG-stimulated RhoA nucleotide binding via a novel fluorescence polarization assay. $J$ Biomol Screen 2009; 14: 161-172.

128 Rusconi P, Caiola E, Broggini M. RAS/RAF/MEK inhibitors in oncology. Curr Med Chem 2012; 19: 1164-1176.

129 Zhao ZS, Manser E. Do PAKs make good drug targets? F1000 Biol Rep 2010; 2: 70.

(1) () $\odot$ This work is licensed under a Creative Commons Attribution-NonCommercial-NoDerivs 3.0 Unported License. To view a copy of this license, visit http:// creativecommons.org/licenses/by-nc-nd/3.0/ 